\title{
Nanoscaled Discotic Liquid Crystal/Polymer Systems: Confinement Effects on Morphology and Thermodynamics
}

\author{
C. Stillings ${ }^{1}$, E. Martin ${ }^{1}$, M. Steinhart ${ }^{2}$, R. Pettau ${ }^{3}$, \\ J. Paraknowitsch ${ }^{1}$, M. Geuss ${ }^{2}$, J. Schmidt ${ }^{1}$, G. Germano ${ }^{1}$, \\ H.-W. Schmidt ${ }^{3}$, U. Gösele ${ }^{2}$, and J. H. Wendorff ${ }^{1}$ \\ ${ }^{1}$ Department of Chemistry, Philipps-Universität Marburg, \\ Marburg, Germany \\ ${ }^{2}$ Max Planck Institute of Microstructure Physics, Halle, Germany \\ ${ }^{3}$ Macromolecular Chemistry I, University of Bayreuth, \\ Bayreuth, Germany
}

We have studied the influence of geometric confinement imposed on the supramolecular architecture of a discotic model compound confined to self-ordered nanoporous alumina. We systematically varied the pore diameter and the chemical nature of the pore walls and studied the systems thus obtained by means of wide angle $X$-ray diffraction and differential scanning calorimetry. A dominant planar core phase was found for high-energy pore walls consisting of alumina, whereas no apparent texture was present in the case of pore walls coated with non-polar poly(p-xylylene). Inside pores of 35 and $180 \mathrm{~nm}$ in diameter, pronounced geometric confinement effects and interfacial effects influence the structure formation. Additionally, we performed molecular dynamics simulations using a coarsegrained discotic potential.

Keywords: coarse-grained molecular dynamics simulation; confinement; discotic liquid crystal; nanopores; thermodynamics

This work was partly supported by the Deutsche Forschungsgemeinschaft (DFG) and the Volkswagenstiftung. The MD simulations were performed in part on the IBM Regatta p690+ parallel computer JUMP of the John von Neumann Institute for Computing in Jülich.

Address correspondence to Christopher Stillings, Department of Chemistry, Philipps-Universität Marburg, Hans-Meerwein-Str., Marburg D-35032, Germany. E-mail: stillings@staff.uni-marburg.de 


\section{INTRODUCTION}

Columnar discotic liquid crystals (LCs) form 1D-stacks exhibiting high mobilities of charge carriers and excitons along their long axes and can therefore be considered as functional LCs. Because of this unique property, discotics are promising materials for applications in opto-electronics. The rational tailoring of their opto-electronic properties may be accomplished very effectively by imposing geometric confinement [1]. We studied two strategies for the nanostructuring of triphenylene-based discotic LCs: template wetting and microphase separation in block copolymer (BC) films. In this report, we will focus on template wetting and modelling of the structure formation of discotics in cylindrical confinement by molecular dynamics simulation (MD).

The controlled generation of supramolecular architectures formed by liquid crystals (LCs) inside ordered nanoporous templates, such as ordered porous alumina [2], is a promising approach towards the integration of functional LCs into device architectures. Nanorods consisting of the discotic LC 2-adamantanoyl-3,6,7,10,11-penta(1-butoxy) triphenylene (AdaPBT) inside ordered nanoporous alumina possess a dominant planar core phase (columns aligned parallel to the pore axes). The surrounding shell is characterised by homeotropic (faceon) anchoring of the molecules and thereby the columns on the pore walls [3]. This structural model is consistent with the results of an exploratory MD simulation employing a coarse-grained discotic potential [4-6].

In the bulk, AdaPBT displays the following phase behaviour [7]: below $75^{\circ} \mathrm{C}$ glassy; above $75^{\circ} \mathrm{C} \mathrm{Col}$ hp (columnar hexagonal-plastic mesophase); above $168^{\circ} \mathrm{C} \mathrm{Col}$ ho (columnar hexagonal-ordered mesophase); above $188^{\circ} \mathrm{C}$ isotropic melt. The $\mathrm{Col}_{\mathrm{hp}}$ phase exhibits an additional correlation between individual columns in the columnar direction. The XRD pattern of AdaPBT [8,9] contains two dominant reflections: the narrow $(100)$ peak at $2 \Theta \approx 5.3^{\circ}(\mathrm{d} \approx 1.6 \mathrm{~nm})$ of both the $\mathrm{Col}_{\text {ho }}$ and the $\mathrm{Col}_{\text {hp }}$ phase related to the inter-columnar center-to-center distance of $\approx 1.9 \mathrm{~nm}$ and a weaker peak at $\approx 25.5^{\circ}(\mathrm{d} \approx 0.35 \mathrm{~nm})$. The latter reflection represents the intra-columnar disk-disk distance and is indexed as (001) in the case of the $\mathrm{Col}_{\text {ho }}$ phase.

\section{EXPERIMENTAL PART}

AdaPBT was synthesized according to the literature [9]. AdaPBT was molten on the surface of native self-ordered porous alumina templates with a pore diameter of $400 \mathrm{~nm}$ and a pore depth of 100 microns (the 
pores have closed pore bottoms) at a temperature of about $230^{\circ} \mathrm{C}$. Subsequently, the samples were quenched by rapid immersion into cold water. In a second series of experiments, self-ordered porous alumina templates of the same type were coated with a $50 \mathrm{~nm}$ thick film of poly(p-xylylene). The coating of the templates was performed in a chemical vapour deposition coating system (Special Coating Systems).

XRD measurements were performed using a Philips X'pert MRD diffractometer with cradle and secondary monochromator for $\mathrm{Cu} \mathrm{K}_{\alpha}$ radiation. The aligned nanowires within the template and the polymer films were oriented parallel to the plane defined by the incident X-ray beam and the detector. Therefore, only the columns oriented parallel to the long axes of the pores (planar phase), or the columns perpendicular to the film surface, respectively, contribute to the detectable intensity of the intra-columnar (001) peak. In turn, only columns oriented perpendicularly to the long axes of the pores (homeotropic phase) or parallel with the film plane, respectively, contribute to the detectable intensity of the inter-columnar (100) reflection. Changes in the intensity ratio between the two peaks thus indicate corresponding changes in the relative ratio of columns having adopted one of these distinct orientations.

Differential scanning calorimetry was performed using a Mettler Toledo calorimeter model ADSC 821e. About $5 \mathrm{mg}$ of the bulk materials were probed using standard aluminium pans. For the confined samples, two alumina templates with a pore diameter of $35 \mathrm{~nm}$ were cut into pieces $8 \mathrm{~mm}$ in diameter, thus precisely matching the sample holders of the calorimeter. Cutting was performed with a computer controlled high-precision milling machine (DMU 50T, Deckel MAHO). An empty membrane piece was used as reference in the DSC measurements.

\section{RESULTS AND DISCUSSION}

\section{Confinement Effects on Structure Formation Processes}

Figure 1 displays the DCS-thermograms of bulk AdaPBT and AdaPBT confined to alumina templates with a pore diameter of $35 \mathrm{~nm}$. It is obvious particularly from the second heating runs that confined AdaPBT exhibits an additional DSC peak that does not appear in the corresponding curves of the bulk sample.

Only a weak shift of the transition temperature from $188^{\circ}$ to $186^{\circ}$ occurs for the transition from the liquid-crystalline to the isotropic state when the AdaPBT is confined to the nanopores, yet intensity and shape of the additional peak indicate an additional phase transition at $179^{\circ} \mathrm{C}$. Obviously, the confinement imposed by the $35-\mathrm{nm}$ pores significantly 


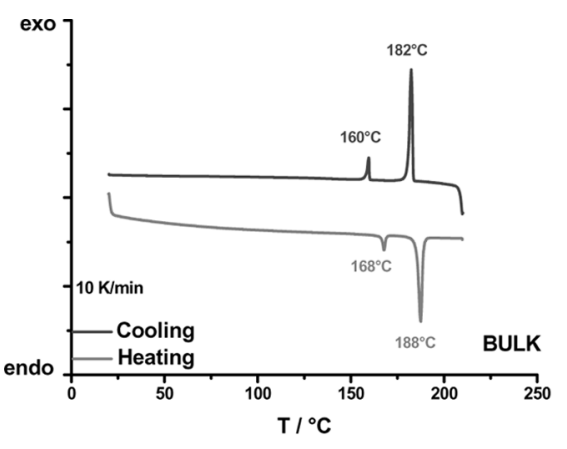

(a)

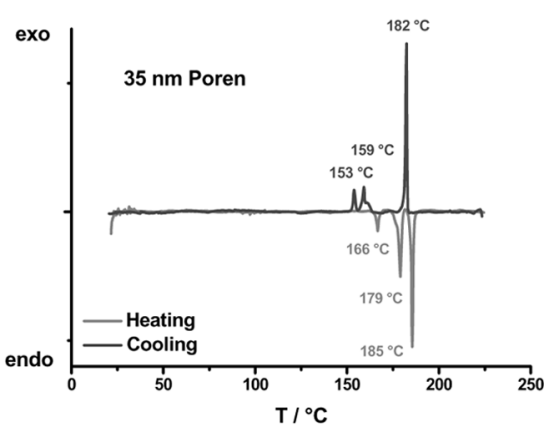

(b)

FIGURE 1 Differential scanning calorimetry diagrams of (a) bulk AdaPBT; (b) AdaPBT confined to self-ordered porous alumina, pore diameter of $35 \mathrm{~nm}$. Heating and cooling rates were $10 \mathrm{~K} / \mathrm{min}$.

influences the structure formation. However, up to now, we could not elucidate the origin of the additional mesophase.

\section{Influence of Pore Walls}

We studied the influence of the polarity of the pore walls on the texture of the mesophase inside the pores. To this end, we infiltrated AdaPBT into pores, the walls of which consisted of alumina and therefore exhibited a high polarity or so to say a high surface energy, as well as into pores with walls coated with a $50 \mathrm{~nm}$-thick non-polar poly(p-xylylene) (PPX) layer thereby presenting pore walls with a much lower surface energy. In both cases, the pore diameter of the native templates used was $400 \mathrm{~nm}$, and that of the templates modified with PPX about $300 \mathrm{~nm}$.

In the non-treated pores, the mesophase shows a pronounced planar texture, as obvious from the high relative intensity of the (001) peak at $2 \Theta \approx 25.5^{\circ}$ (Fig. 2a). In the case of the templates coated with PPX, the XRD pattern corresponds to that of a powder sample and no apparent texture can be found (Fig. 2b). This indicates that polar pore walls having high surface energy enable the formation of a dominant planar phase.

According to our structural model deduced from XRD analysis of AdaPBT in alumina pores, the discotic arrangement consists of an ordered planar core phase surrounded by a homeotropic shell characterised by poor ordering [3]. Obviously, the confinement imposed by the template pores has an effect on the mesophase structure and its formation. Besides the pore diameter and the pore surface, 


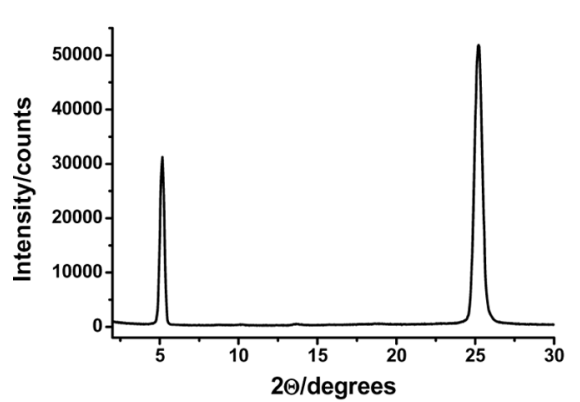

(a)

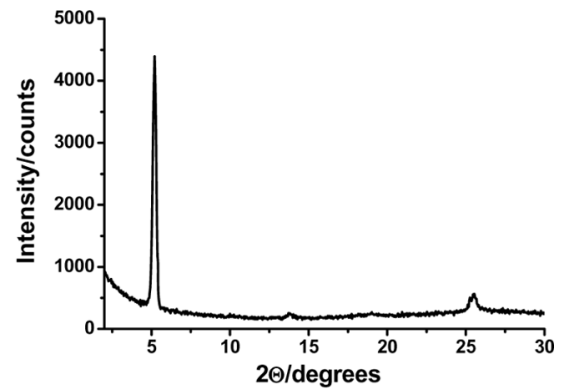

(b)

FIGURE 2 Wide angle XRD scans of aligned AdaPBT nanorods inside porous alumina with a pore diameter of $400 \mathrm{~nm}$. (a) Pore walls consist of alumina; (b) pore walls are coated with a $50 \mathrm{~nm}$ thick PPX layer.

a set of other parameters should influence the structure formation process, such as the composition of the discotic LC, the curvature of the pore walls and the temperature profile applied to the samples during structure formation. In order to understand the formation process on a molecular scale and to quantify the influence of the different parameters on the resulting mesophase structure and the formation process, MD simulations were performed.

\section{Molecular Dynamics Simulations}

While there are many computer simulations of confined systems in slab geometry (also called a "slit" pore), see, e.g. recent work by Piñeiro et al. [10] and references contained therein, simulations in cylindrical geometry are rare, and so far none of them have been performed with liquid crystals. The large amount of molecules needed makes it convenient to do the modelling at molecular rather than atomic detail even when resorting to massive parallel computing. Thus we approximated discotic mesogens with soft rigid oblate ellipsoids interacting with the Bates-Luckhurst extension [4] from prolate to oblate shapes of the Gay-Berne angular-dependent potential [11], that can be considered as an anisotropic generalisation of the 12-6 Lennard-Jones potential:

$$
\begin{aligned}
U_{i j}\left(\mathbf{r}_{i j}, \hat{\mathbf{e}}_{i}, \hat{\mathbf{e}}_{j}\right)=4 \varepsilon\left(\hat{\mathbf{r}}_{i j}, \hat{\mathbf{e}}_{i}, \hat{\mathbf{e}}_{j}\right)\{ & {\left[\frac{r_{i j}-\sigma\left(\hat{\mathbf{r}}_{i j}, \hat{\mathbf{e}}_{i}, \hat{\mathbf{e}}_{j}\right)+\sigma_{\mathrm{ff}}}{\sigma_{\mathrm{ff}}}\right]^{-12} } \\
& \left.-\left[\frac{r_{i j}-\sigma\left(\hat{\mathbf{r}}_{i j}, \hat{\mathbf{e}}_{i}, \hat{\mathbf{e}}_{j}\right) \sigma_{\mathrm{ff}}}{\sigma_{\mathrm{ff}}}\right]^{-6}\right\} .
\end{aligned}
$$


Here $\varepsilon$ and $\sigma$ are orientation-dependent energy and shape functions; $\mathbf{r}_{i j}=r_{i j} \hat{\mathbf{r}}_{i j}$ is the intermolecular separation; $\hat{\mathbf{e}}_{i}$ and $\hat{\mathbf{e}}_{j}$ are the molecular symmetry axes; $\sigma_{\mathrm{ff}}=\xi \sigma_{0}$ with $\xi=0.2$ is the molecular thickness or face-to-face separation, and $\sigma_{\mathrm{ee}}=\sigma_{0}$ is the molecular diameter or edge-to-edge separation. For the molecule-molecule interaction we used a parameter set that reproduces approximately the properties of a substance like hexakis(butoxy)triphenylene: $\mu=1, \nu=2, \kappa=0.2$, $\kappa^{\prime}=0.1[5]$. We neglected the adamantanoyl substituent because the coarse-grained modelling of an axially symmetric molecule is far easier.

For the molecule-surface interaction, only potentials in slab geometry have been developed so far, for spherical particles [12] as well as for prolate [13] and oblate [6] shapes. The reason is that finding the exact mesoscopic functional form of the moleculesurface interaction potential for systems confined in a cylindrical geometry is not as easy as in the slab case: the integral over an infinitely thick region outside a cylinder of radius $R$ opposed to the integral of an infinite half-space is awkward even for LennardJones atoms, leading to elliptic type integrals and hypergeometric functions [14]. On the other hand, since in our exploratory run we used about 97000 molecules confined in a cylinder with a diameter $2 R=35 \sigma_{0}$, we felt it acceptable to neglect the curvature in a preliminary exploration. The important feature of the surface interaction is to favour homeotropic (face-on) anchoring, which is achieved by the Bellier-Castella potential [6] employed by us. The latter depends on the molecule-wall separation $r_{i \mathrm{w}}$ and the angle $\theta_{i \mathrm{w}}$ between the molecular symmetry axis $\hat{\mathbf{e}}_{i}$ and the normal to the wall:

$$
\begin{aligned}
U_{i \mathrm{w}}\left(r_{i \mathrm{w}}, \theta_{i \mathrm{w}}\right)=\varepsilon_{\mathrm{w}}\left[1+A P_{2}\left(\cos \theta_{i \mathrm{w}}\right)\right]\{ & \frac{2}{15}\left[\frac{r_{\mathrm{w}}-r_{\text {shift }}\left(\theta_{i \mathrm{w}}\right)}{\sigma_{\mathrm{ff}}}\right]^{-9} \\
& \left.-\left[\frac{r_{i \mathrm{w}}-r_{\text {shift }}\left(\theta_{i \mathrm{w}}\right)}{\sigma_{\mathrm{ff}}}\right]^{-3}\right\} .
\end{aligned}
$$

Two simulated systems are displayed in Figure 3, with the molecules coloured depending on their orientation with respect to the cylinder axis, using the molecular graphics program for convex body fluids QMGA [15]. In spite of the simplifications, the simulation results agree extraordinarily well with the experimental results obtained for AdaPBT confined inside nanoporous alumina. According to our structure model deduced from XRD analysis, there is an ordered planar 

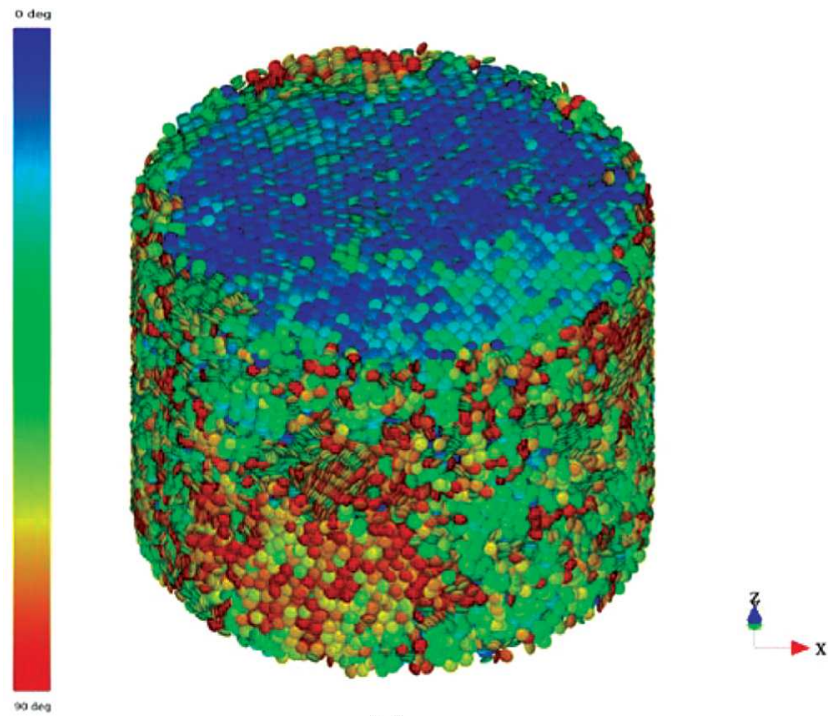

(a)
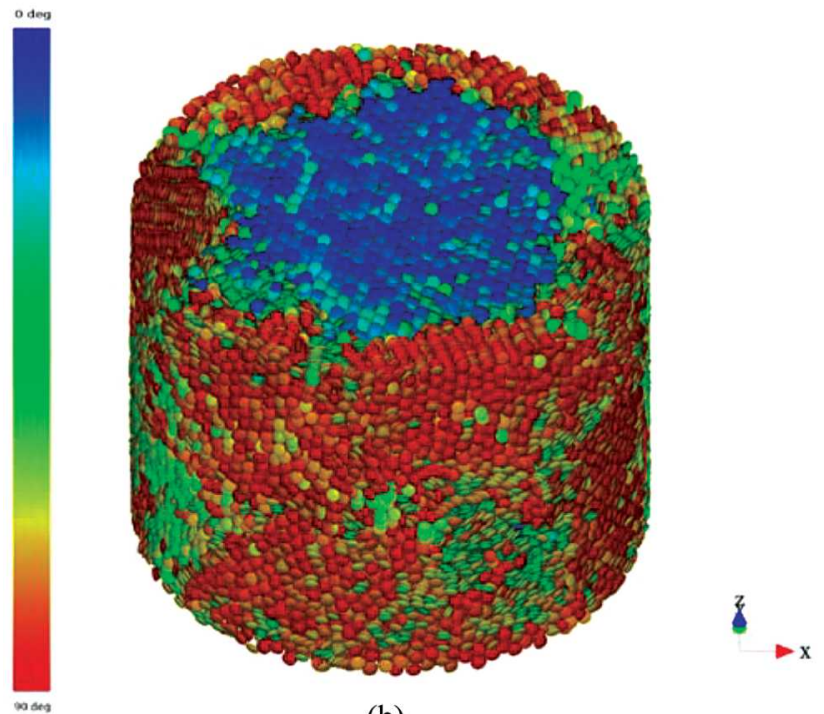

(b)

FIGURE 3 Snapshots from MD runs in the canonic ensemble at $\mathrm{T}^{*}=9.0$ and $\rho^{*}=3.8$, with an observed $\mathrm{P}^{*} \approx 33$. The particle-wall interaction strength $\varepsilon_{\mathrm{W}}$ has the value 14 for (a) and 30 for (b). 
core phase with columnar hexagonal structure (in blue) surrounded by a homeotropic shell characterized by poor ordering (in other colours); the thickness of the shell increases with the strength of the molecule-wall interaction potential [3].

Obviously by increasing the interaction intensity of the molecules with the pore wall, the extent of the homeotropic phase is also increasing. This shows the strong effect of the nature of the pore surface on the mesophase structure inside the pores just as seen in the experiment. In addition, it can be observed that by increasing the molecule surface interaction the molecules are attached flatter on the pores surface.

Though our simulations so far disregard surface curvature and intramolecular detail, they agree extraordinarily well with the experimental results obtained for AdaPBT nanorods inside porous alumina. As shown, the results of the simulations are consistent with the experimental findings and will enable a more quantitative analysis of the experimentally identified parameters relevant to structure formation.

\section{CONCLUSION AND OUTLOOK}

Two types of strategies applied by us, template wetting and microphase separation in LC block copolymers, which will be reported in detail elsewhere [16], impose strong confinement on the discotic mesophases and modify their structural and thermodynamic properties. Modifications of the polarity of the pore walls, guided by MD simulations of the structure formation processes, allow engineering the supramolecular architecture of LC nanorods and thereby their optical and electronic properties. In case of the MD simulations, it is our aim to develop a specific potential for cylindrical geometries to further improve the surprisingly good results obtained until now. Furthermore, additional series of experiments and simulations with different pore diameters, pressures, temperatures and intermolecular interactions will be performed. Future experiments and MD simulations should contribute to a fundamental understanding of the structure formation processes in confined systems.

\section{REFERENCES}

[1] Kopitzke, J., Wendorff, J. H., \& Glüsen, B. (2000). Liquid Cryst., 27, 643.

[2] Masuda, H. \& Fukuda, K. (1995). Science, 268, 1466. 
[3] Steinhart, M., Zimmermann, S., Göring, P., Schaper, A. K., Gösele, U., Weder, C., \& Wendorff, J. H. (2005). Nano Lett., 5, 429.

[4] Bates, M. A. \& Luckhurst, G. R. (1996). J. Chem. Phys., 104, 6696.

[5] Caprion, D., Bellier-Castella, L., \& Ryckaert, J.-P. (2003). Phys. Rev. E, 67, 041703.

[6] Bellier-Castella, L., Caprion, D., \& Ryckaert, J.-P. (2004). J. Chem. Phys., 121, 4874.

[7] Bayer, A., Kopitzke, J., Noll, F., Seifert, A., \& Wendorff, J. H. (2001). Macromolecules, 34, 3600 .

[8] Glüsen, B., Kettner, A., \& Wendorff, J. H. (1997). Mol. Cryst. Liq. Cryst., 303, 115.

[9] Kettner, A. \& Wendorff, J. H. (1999). Liquid Cryst., 26, 483.

[10] Piñeiro, M. M., Galindo, A., \& Parry, A. O. (2007). Soft Matter, 3, 768.

[11] Gay, J. G. \& Berne, B. J. (1981). J. Chem. Phys., 74, 3316.

[12] Steele, W. A. (1973). Surf. Sci., 36, 317.

[13] Wall, G. D. \& Cleaver, D. J. (1997). Phys. Rev. E, 56, 4306.

[14] Jiang, G., Zhang, J., Zhang, X., \& Wang, W. (2004). ANZIAM J., 46, E70.

[15] Gabriel, A. T., Meyer, T., \& Germano, G. (2008). J. Chem. Theory Comput., 4, 468.

[16] Stillings, C., et al., in preparation. 